\title{
No serological evidence of Rickettsia helvetica infection in Scandinavian sarcoidosis patients
}

\author{
A. Planck*, A. Eklund*, J. Grunewald*, S. Vene $\#$,
}

No serological evidence of Rickettsia helvetica infection in Scandinavian sarcoidosis patients. A. Planck, A. Eklund, J. Grunewald, S. Vene. (C) ERS Journals Ltd 2004.

ABSTRACT: Many agents have been suggested to elicit sarcoidosis, and, recently, an association was presented between this disease and the bacterium Rickettsia helvetica.

The aim of this study was to investigate if serological support for such an association could be detected.

Sera from 20 well-characterised sarcoidosis patients were investigated for antirickettsial immunoglobulin $G$ antibodies with a micro-immunofluorescence technique. $R$. helvetica, $R$. conorii and $R$. typhi served as antigens.

In conclusion, none of the investigated sera displayed detectable titres of antirickettsial immunoglobulin $\mathbf{G}$ antibodies. Thus, the current study does not support an association between rickettsia and sarcoidosis.

Eur Respir J 2004; 24: 811-813.
*Dept of Medicine, Division of Respiratory Medicine, Karolinska Hospital, ${ }^{\#}$ Swedish Institute for Infectious Disease Control, and Microbiology and Tumour Biology Centre, Karolinska Institutet, Stockholm, Sweden.

Correspondence: A. Planck, Dept of Medicine, KSS, S-541 85 Skövde, Sweden.

Fax: 46500432554

E-mail: anders.planck@vgregion.se

Keywords: Antibody, bacteria, infection, Rickettsia helvetica, sarcoidosis

Received: February 42004

Accepted after revision: June 132004

This study was supported by the King Oscar II Jubilee foundation, the Swedish Research Council and the Karolinska Institutet. Purified $R$. helvetica antigen was kindly provided by D. Raoult (Unité des Rickettsies, Faculté de Medecine, Marseille, France). R. conorii and $R$. typhi antigens were a gift from M.G. Peacock (Rocky Mountain Laboratories, Hamilton, MT, USA).
Although the understanding of the inflammatory mechanisms in sarcoidosis has increased during the last decades, the cause of the disease remains an enigma. One hypothesis is that an infectious agent elicits the disease and numerous candidates have been proposed [1, 2]. Among these candidates, mycobacterial species are probably the most frequently suggested. Their association with sarcoidosis is based upon histological similarities between sarcoidosis and mycobacterial infections and findings of mycobacterial residues or genetic material in sarcoid tissue [2, 3]. Mycobacterial species have, however, never been successfully isolated and cultured from sarcoid tissue.

In 2002, a new candidate agent, Rickettsia helvetica, was suggested when NiLSSON et al. [4] found genetic material from these bacteria in patients diagnosed with sarcoidosis.

$R$. helvetica belongs to the spotted fever group (SFG) of rickettsiae. Members of this group share several antigenic characteristics, making cross-reactions between SFG species common, and they are usually spread to vertebrates by ticks. Speculations that sarcoidosis is a disease spread by ticks have previously been presented. Based upon clinical similarities with Lyme disease and high levels of antibodies against Borrelia burgdorferi in Chinese sarcoidosis patients, this tick-borne bacterium was suggested as a trigger of sarcoidosis [5, 6]. However, later attempts to repeat these results have failed [7, 8].

The aim of this study was to find further evidence for the suggested association between sarcoidosis and $R$. helvetica by investigating the immune response against rickettsial species in well-characterised sarcoidosis patients.

\section{Materials and methods}

Study subjects

A total of 20 patients with sarcoidosis (median age $38 \mathrm{yrs}$ range $28-62$ yrs; 13 males and seven females) participated in the study. All of the patients revealed a clinical picture that was compatible with sarcoidosis and they were considered as having active disease [9]. The diagnosis was based upon histopathological evidence of non-caseating granulomas $(n=12)$ and/or Löfgren's syndrome $(n=11)$, i.e. acute onset with fever, erythema nodosum and/or ankle arthritis and the presence of bilateral hilar adenopathy [1]. Sera from all of the patients with Löfgren's syndrome and from three patients with more insidious onset of symptoms, e.g. dry cough, low-grade fever and fatigue, were analysed at the time of diagnosis. Sera from the remaining six patients were collected after a disease duration of $\geqslant 2$ yrs (table 1$)$. In addition, sera were also analysed from nine of the included patients after clinical and radiological resolution of disease.

\section{Methods}

A micro-immunofluorescence (MIF) test was employed to investigate the presence of specific anti-rickettsial immunoglobulin $(\mathrm{Ig}) \mathrm{G}$ antibodies in patient sera [10]. R. conorii and $R$. typhi were used as control antigens for species belonging to the SFG and typhus groups of rickettsiae, respectively. Sera 
Table 1. - Chest radiograph classification at the time of serum sampling (disease onset or $\geqslant 2$ yrs after diagnosis)

\begin{tabular}{|c|c|c|c|}
\hline \multirow{2}{*}{$\begin{array}{l}\text { Chest } \\
\text { radiograph }\end{array}$} & \multicolumn{2}{|c|}{ Disease onset } & \multirow{2}{*}{$\begin{array}{l}\text { Disease } \\
\geqslant 2 \text { yrs }\end{array}$} \\
\hline & $\begin{array}{c}\text { Acute onset } \\
\text { (Löfgren's syndrome) }\end{array}$ & $\begin{array}{l}\text { Insidious } \\
\text { onset }\end{array}$ & \\
\hline
\end{tabular}

\begin{tabular}{llll}
\hline Stage 0 & 0 & 0 & 1 \\
Stage I & 7 & 3 & 1 \\
Stage II & 4 & 0 & 2 \\
Stage III & 0 & 0 & 2 \\
\hline
\end{tabular}

Data are presented as $\mathrm{n}$. Patients investigated at disease onset are subdivided depending on initial symptoms. Stage 0: normal chest radiograph; stage I: bilateral hilar adenopathy (BHL) without parenchymal shadowing; stage II: BHL with parenchymal shadowing; stage III: parenchymal shadowing without BHL.

from patients diagnosed with spotted fever or murine typhus served as positive controls with end-point IgG titres of 640 and 1,280 for $R$. conorii and $R$. typhi, respectively.

\section{Results}

In contrast to positive controls, all sera from sarcoidosis patients, irrespective of clinical stage, were negative for detectable antibodies to Rickettsia species.

\section{Discussion}

In the present study, none of 20 well-characterised sarcoidosis patients revealed serological signs of rickettsial infection. The result does not support the recent study by NILSSON et al. [4], in which a strong association between sarcoidosis and signs of $R$. helvetica infection was suggested.

The genus Rickettsia includes Gram-negative, obligate intracellular bacteria, which are transmitted to humans by arthropods [11]. Several species are potentially harmful to humans, as exemplified by $R$. prowazekii and $R$. rickettsii causing epidemic typhus and Rocky Mountain spotted fever, respectively, whilst others are not known to cause disease in humans. However, the number of known rickettsial species that are pathogenic to humans is increasing and has almost doubled during the last decades. In Sweden, human Rickettsia diseases are uncommon and, in principal, restricted to a few import cases each year. As a consequence, the Swedish seroprevalence of Rickettsia antibodies is very low.

$R$. helvetica was first isolated in Switzerland from Ixodes ricinus ticks in 1979 [12] and has since been isolated from the same tick in other European countries, such as France [13] and Sweden [14]. Although I. ricinus commonly bites humans, the pathogenic potential for $R$. helvetica remains somewhat unclear and is restricted to a few case descriptions. NILSSON et al. [15] previously reported signs of $R$. helvetica infection in two cases of sudden death in young males with myocarditis. In a French sero-survey by FoURNIER et al. [16], 9.2\% of 379 forest workers were seropositive to $R$. helvetica. The authors also detected an elevation in antibody titres against $R$. helvetica in a patient presenting with transient symptoms of fever, fatigue and myalgias, suggesting an ongoing infection by this bacterium.

The association between $R$. helvetica and sarcoidosis presented by NILSSON et al. [4] was based upon the detection of genetic material by PCR technique in two patients with sarcoidosis that was diagnosed post mortem (one patient was previously reported as a case of sudden death in the study by NiLSSON et al. [15]).
Furthermore, immunohistochemical signs consistent with rickettsial infection were detected in archival sarcoid tissues from 26 out of 30 patients. Together with electron microscopy findings in tissues from a few patients, this was suggested to support an association between $R$. helvetica and sarcoidosis. However, NILSSON et al. [4] did not succeed in isolating any microorganism, nor did they analyse serum for antibody titres against any Rickettsia species.

Most of the patients in the current study came from a similar geographical area in Sweden as the patients studied by NiLSSON and co-workers [4]. It should also be noted that almost every patient who was immunohistochemically studied by NILSSON et al. [4] was reported to exhibit evidence of rickettsial infection. Thus, it is unlikely that the failure to find serological support for rickettsial infection in the current patients is explained by differences in patient populations. The risk of excluding relevant subgroups was minimised by including patients with differences in clinical appearance.

In this study, the well-established MIF technique was relied upon for demonstration of an ongoing infection with rickettsia. The antibody production in rickettsial infections is characterised by an early IgM response followed by an increase in IgG titres. Exceptions from this pattern do occur, since human $R$. prowazekii infections may exist in a latent form for several decades until reactivation and presentation as Brill-Zinsser disease [17]. However, although the antibody response in this condition diminishes during the latency phase, it characteristically returns when the infection reactivates. It should also be noted that an activation of the humoural response with hypergammaglobulinaemia is considered to be a prominent feature of sarcoidosis. Thus, knowledge of the common serological response in established rickettsioses, as well as the immune response in sarcoidosis, contradicts a rickettsial infection in the current patients.

In conclusion, in the present study of well-characterised Scandinavian sarcoidosis patients with different patterns and duration of disease, none had a serological response against Rickettsia species. The results do not support the hypothesis that Rickettsia helvetica is involved in the aetiology of sarcoidosis.

Acknowledgements. The authors are grateful for assistance by B. Dahlberg.

\section{References}

1. Joint Statement of the American Thoracic Society (ATS), the European Respiratory Society (ERS) and the World Association of Sarcoidosis and Other Granulomatous Disorders (WASOG) adopted by the ATS Board of Directors and by the ERS Executive Committee. Statement on sarcoidosis. Am J Respir Crit Care Med 1999; 160: 736755.

2. du Bois RM, Goh N, McGrath D, Cullinan P. Is there a role for microorganisms in the pathogenesis of sarcoidosis? J Intern Med 2003; 253: 4-17.

3. Mangiapan G, Hance AJ. Mycobacteria and sarcoidosis: an overview and summary of recent molecular biological data. Sarcoidosis 1995; 12: 20-37.

4. Nilsson K, Pahlson C, Lukinius A, Eriksson L, Nilsson L, Lindquist O. Presence of Rickettsia helvetica in granulomatous tissue from patients with sarcoidosis. J Infect Dis 2002; 185: $1128-1138$

5. Jacob F. Could Borrelia burgdorferi be a causal agent of sarcoidosis? Med Hypotheses 1989; 30: 241-243.

6. Hua B, Li QD, Wang FM, Ai CX, Luo WC. Borrelia 
burgdorferi infection may be the cause of sarcoidosis. Chin Med J (Engl) 1992; 105: 560-563.

7. Arcangeli G, Calabro S, Cisno F, Zambotto FM, Drigo R, Ferraresso A. Determination of antibodies to Borrelia burgdorferi in sarcoidosis. Sarcoidosis 1994; 11: 32-33.

8. Martens H, Zöllner B, Zissel G, Burdon D, Schlaak M, Müller-Quernheim J. Anti-Borrelia burgdorferi immunoglobulin seroprevalence in pulmonary sarcoidosis: a negative report. Eur Respir J 1997; 10: 1356-1358.

9. Consensus conference: activity of sarcoidosis. Third WASOG meeting, Los Angeles, USA, September 8-11, 1993. Eur Respir J 1994; 7: 624-627.

10. Philip RN, Casper EA, Ormsbee RA, Peacock MG, Burgdorfer W. Microimmunofluorescence test for the serological study of Rocky Mountain spotted fever and typhus. J Clin Microbiol 1976; 3: 51-61.

11. Raoult D, Roux V. Rickettsioses as paradigms of new or emerging infectious diseases. Clin Microbiol Rev 1997; 10 : 694-719.
12. Burgdorfer W, Aeschlimann A, Peter O, Hayes SF, Philip RN. Ixodes ricinus: vector of a hitherto undescribed spotted fever group agent in Switzerland. Acta Trop 1979; 36: $357-$ 367.

13. Parola P, Beati L, Cambon M, Raoult D. First isolation of Rickettsia helvetica from Ixodes ricinus ticks in France. Eur J Clin Microbiol Infect Dis 1998; 17: 95-100.

14. Nilsson K, Lindquist O, Liu AJ, Jaenson TG, Friman G, Pahlson C. Rickettsia helvetica in Ixodes ricinus ticks in Sweden. J Clin Microbiol 1999; 37: 400-403.

15. Nilsson K, Lindquist O, Pahlson C. Association of Rickettsia helvetica with chronic perimyocarditis in sudden cardiac death. Lancet 1999; 354: 1169-1173.

16. Fournier PE, Grunnenberger F, Jaulhac B, Gastinger G, Raoult D. Evidence of Rickettsia helvetica infection in humans, eastern France. Emerg Infect Dis 2000; 6: 389392.

17. Lutwick LI. Brill-Zinsser disease. Lancet 2001; 357: 11981200 . 